\title{
Coronary reserve in patients with aortic valve disease before and after successful aortic valve replacement
}

\author{
F. R. Eberli, M. Ritter, J. Schwitter, A. Bortone, J. Schneider, O. M. Hess and H.-P. Krayenbuehl \\ Medical Policlinic, Division of Cardiology, University Hospital, Zurich, Switzerland
}

KEY WORDS: Coronary vasodilator capacity, aortic valve disease, left ventricular hypertrophy, regression of muscle mass.

In patients with aortic valve disease and normal coronary angiograms coronary reserve was determined by the coronary sinus thermodilution technique. Three groups of patients were studied: 37 preoperative patients; 18 different patients 12-52 months after aortic valve replacement and seven control subjects with no cardiac disease. Coronary flow ratio (dipyridamole/rest) was diminished in preoperative compared with postoperative patients ( $1.66 \pm 0.44$ vs $2.22 \pm 0.85$; $\mathrm{P}<0.05)$ as well as with controls $(2.80 \pm 0.84 ; \mathrm{P}<0.01)$, and corresponding coronary resistance ratio (dipyridamole) rest) was higher in preoperative patients than in both other groups $(0.61 \pm 0.17$ vs $0.48 \pm 0 \cdot 14 ; \mathrm{P}<0.05$ vs $0.37 \pm 0.10$; $\mathrm{P}<0.01)$. Differences in the flow ratio, but not in the resistance ratio, were significant $(\mathbf{P}<0.05)$ in patients after aortic valve replacement compared with controls. Total coronary sinus blood flow at rest was elevated in preoperative compared with both postoperative patients and controls $\left(252 \pm 99\right.$ vs $169 \pm 63 ; \mathrm{P}<0.01 ;$ vs $\left.170 \pm 35 \mathrm{ml}^{\mathrm{min}} \mathrm{m}^{-1}, \mathrm{P}<0.05\right)$, whereas

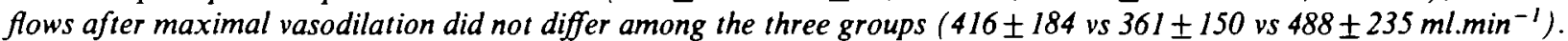
Postoperative patients showed a distinct, though not total regression of left ventricular angiographic muscle mass index and wall thickness. Nine of the 18 postoperative patients showed a normal coronary flow reserve and nine showed subnormal response. These two subgroups did not differ with respect to preoperative macroscopic and microscopic measures of hypertrophy.

Thus in aortic valve disease, the reduced coronary vasodilator capacity is mainly due to an elevated coronary flow at rest, while the maximal coronary blood flow achieved is identical to that of postoperative patients and controls. With regression of left ventricular hypertrophy, flow at rest decreases and this leads to a distinct improvement of coronary flow reserve.

\section{Introduction}

Left ventricular hypertrophy induced by chronic pressure and volume overload is associated with a reduced coronary flow reserve and vasodilator capacity ${ }^{[1-8]}$. These abnormalities of coronary dynamics are thought to be major reasons for the occurrence of angina pectoris in patients with aortic stenosis or insufficiency and normal coronary arteries $^{[9,10]}$. Various mechanisms alone or in combination may lead to the reduced coronary flow reserve, as assessed by the ratio of maximally vasodilated and autoregulated basal flow. Maximal coronary flow may be depressed in hypertrophy as a consequence of a reduction of the vascular bed either functional, from extravascular compressive forces or structural, from excess interstitial collagen formation with intercellular scarring ${ }^{[1,12]}$. Alternatively, coronary flow reserve may be decreased in hypertrophy due to an increased flow at rest with unchanged maximally achieved flow ${ }^{[13-19]}$. This encroachment on flow reserve by vasodilation at rest becomes necessary because growth of the vessels throughout the vascular tree does not keep pace with the increase in ventricular mass ${ }^{[16]}$

Submitted for publication on 9 November 1989, and in revised form 18 April 1990.

This work was supported by a grant of the Swiss Foundation of Cardiology.

Address for reprints: Hans Peter Krayenbuehl, MD, Chief of Cardiotogy, Medical Poticlinic, University Hospilal, Racmirts. 100, CH-8091 Zurich, Switzerland.
It has been shown ${ }^{[17]}$ that a normal coronary flow reserve can be restored in hypertensive rats by reversal of left ventricular hypertrophy. In man it has been established that regression of left ventricular hypertrophy occurs following removal of an abnormal haemodynamic burden by valve replacement ${ }^{[18,19]}$. However, it is not known whether coronary flow reserve and vasodilator capacity improve postoperatively as well.

Thus, it was the purpose of this study to investigate in patients with aortic valve disease and left ventricular hypertrophy whether (1) such improvements in coronary flow reserve and vasodilator capacity do occur after valve replacement and (2) maximal vasodilated flow is maintained after regression of left ventricular hypertrophy. No reduction in maximal coronary flow would imply that the luminal cross-sectional surface of the resistance vessels remained uninfluenced by the regression process of the hypertrophied myocardium.

\section{Methods}

\section{PATIENT POPULATION}

Three groups of patients were studied. At cardiac catheterization all patients had a normal coronary angiogram and were in sinus rhythm. Patients with aortic valve disease included in this study had no other concomitant valve disease. The preoperative patient group consisted of 37 patients (nine women, 28 men; mean age $57 \pm 10$ years) 
who underwent cardiac catheterization for preoperative evaluation of aortic valve disease, 28 of 37 presented with predominant aortic stenosis, nine of 37 with predominant aortic insufficiency. Ten patients with combined aortic valve disease were classified as having aortic stenosis $(\mathrm{n}=$ 6) when aortic valve area was $\leqslant 1.0 \mathrm{~cm}^{2}$ and as having aortic insufficiency $(n=4)$ when the aortic valve area was $>1.0 \mathrm{~cm}^{2}$. The postoperative patient group consisted of 18 patients (one women, 17 men, mean age $58 \pm 8$ years) who were studied $12-52$ months (mean 30 months) after successful aortic valve replacement. None were included in the preoperative patient group. Aortic valve replacement was carried out in 11 patients for aortic stenosis, in seven for aortic insufficiency. Seven patients received a mechanical prosthesis, 11 patients a bioprosthesis. Postoperative patients selected for study were those followed in this hospital and had their original (i.e. preoperative) cardiac catheterization at this institution. All patients demonstrated a good postoperative result without haemodynamic compromise. The residual pressure gradient over the aortic prosthesis was assessed by CW-Doppler echocardiography in 16 of the 18 patients. It ranged from 4 to $36 \mathrm{~mm} \mathrm{Hg}$ with a mean value of $18.7 \pm 7.4 \mathrm{mmHg}$. Patients agreed to undergo right heart catheterization on a voluntary basis. The control group comprised seven patients (two women, five men; mean age $50 \pm 10$ years) evaluated for atypical chest pain. During upright bicycle exercise testing ST-segment depression of more than $0.1 \mathrm{mV}$ was found in four of these patients (two women, two men; range $0.12-0.35 \mathrm{mV}$ ), but all patients showed a normal physical working capacity of $97-104 \%$ of the age-, sex and height-corrected normal value. Cardiac catheterization revealed no cardiac disease, in particular normal coronary arteries. However, no ergonovine test was performed. Informed consent was obtained from all patients under a protocol approved by the Human Studies Committee of the University Hospital.

\section{CARDIAC CATHETERIZATION}

All cardiac medications were withheld for $12-24 \mathrm{~h}$ before catheterization. Premedication consisted of $10 \mathrm{mg}$ chlordiazepoxide (Librium ${ }^{\circledR}$ ) given orally $1 \mathrm{~h}$ before the procedure. The preoperative patients and the controls underwent right and left heart catheterization. Aortic pressure was measured through a fluid-filled $8 \mathrm{~F}$ pigtail catheter introduced through the right femoral artery. In patients with aortic valve disease left ventricular pressure was obtained with a transseptally introduced $8.5 \mathrm{~F}$ Brockenbrough catheter. Right-sided pressures and pulmonary capillary wedge pressure were measured with a $7 \mathrm{~F}$ Cournand catheter introduced through the right femoral vein. In control patients right-sided pressures, but not pulmonary capillary wedge pressure were obtained using a $6 \mathrm{~F}$ pacing catheter with a lumen for pressure recordings. A peripheral lead of the standard electrocardiogram was recorded together with the pressure tracings. Left ventricular cineangiography was performed in the right and left anterior oblique projection. $40-50 \mathrm{ml}$ of the non-ionic cardiographic contrast iopamidol (755.2 mg.ml ${ }^{-1}$, trometamol $1 \mathrm{mg} \cdot \mathrm{ml}^{-1}=$ lopamiro $370^{\circ}$ ) was injected at a flow rate of $12 \mathrm{ml} \mathrm{s}^{-1}$. In the postoperative patient group an ambulatory right heart catheterization was performed. After the measurement of right-sided pressures with a $7 \mathrm{~F}$ Cournand catheter, an $8 \mathrm{~F}$ pigtail catheter was introduced in the pulmonary artery. Biplane left ventricular angiography was obtained by injection of radiographic contrast into the pulmonary artery $(55 \mathrm{ml}$ at a flow rate of $18-20 \mathrm{ml} \mathrm{s}^{-1}$ ). With this technique a left ventricular opacification was obtained of sufficient quality for quantitative assessment of left ventricular volumes and wall thickness.

Left ventricular volumes were calculated by the arealength method ${ }^{[20]}$ and left ventricular muscle mass according to the method of Rackley et al. ${ }^{[2]]}$. Assuming a thin-walled left ventricular ellipsoid model, end-diastolic circumferential wall stress was calculated according to the formula of Sandler and Dodge ${ }^{[22]}$ and peak systolic wall stress according to the method of Gaasch et al. ${ }^{[23]}$. Aortic regurgitant fraction was determined by the thermodilution technique or by comparison of cardiac output calculated by means of angiography and the Fick method. Values reported are values of thermodilution or a verage of both methods. In postoperative patients arterial pressure was measured with an arm sphygmomanometer and systolic cuff pressure was used instead of left ventricular systolic pressure for calculation of systolic wall stress. Left ventricular end-diastolic pressure was substituted by mean pulmonary capillary wedge pressure for calculation of end-diastolic stress in this latter patient group.

\section{CORONARY BLOOD FLOW MEASUREMENTS}

Measurement of coronary sinus blood flow was performed following the diagnostic catheterization in all preoperative patients and three controls. Four controls were studied on an ambulatory basis with right heart catheterization, at a mean of 10 days after diagnostic coronary arteriography. Ventriculography was not repeated in these four patients and therefore left ventricular volumes and muscle mass reported are the values obtained at the diagnostic catheterization. Total coronary blood flow was measured by the coronary sinus thermodilution technique $^{[24]}$. A 7F thermodilution catheter (CCS-7U-90 A or B, Webster Laboratory, Altadena, CA) was introduced from the right femoral or in a few cases from the right antecubital vein and advanced $2 \mathrm{~cm}$ into the coronary sinus. Correct positioning was checked by measuring oxygen saturation and by injection of small amounts of contrast dye before and after measurements and documented by angiography. Injection of cold saline into the superior vena cava was not associated with changes in the temperature curve of the thermistor in the coronary sinus $^{[2]}$. The signals of the external (mixing temperature of blood and saline) and internal (temperature of the injected saline) thermistors were recorded on an 'Electronics for Medicine VR-12' oscillograph at a paper speed of $5 \mathrm{~mm} \cdot \mathrm{s}^{-1}$. Saline at room temperature was infused through the thermodilution catheter at a rate of $50 \mathrm{ml} \mathrm{min}-1$ and coronary sinus blood flow (CSBF; ml.min ${ }^{-1}$ ) was calculated according to the formula of 
Ganzet al. ${ }^{[24)}$. Coronary resistance (CR; mmHg.min.ml $\mathrm{m}^{-1}$ ) was calculated according to the following equation:

$$
\mathrm{CR}=(\mathrm{MAP}-\mathrm{CSP}) / \mathrm{CSBF}
$$

where $\mathrm{MAP}=$ mean aortic pressure $(\mathrm{mmHg}), \mathrm{CSP}=$ mean coronary sinus pressure $(\mathrm{mmHg})$. Normalization per $100 \mathrm{~g}$ left ventricular muscle was carried out for coronary sinus blood flow and coronary resistance using angiographic mass. In the preoperative patients and in three controls, mean aortic pressure was determined invasively, whereas in postoperative patients and four controls in whom separate right heart catheterization was performed, mean aortic pressure was calculated as diastolic cuff pressure plus $33 \%$ of pulse pressure amplitude. Coronary sinus blood flow was determined at rest and after infusion of $0.5 \mathrm{mg} . \mathrm{kg}^{-1}$ body weight dipyridamole over $15 \mathrm{~min}$. This duration of infusion of dipyridamole was chosen in order to minimize the systemic effects of dipyridamole on heart rate and blood pressure ${ }^{(26)}$. In no case were adverse side-effects of dipyridamole encountered.

Coronary flow ratio (CFR) or coronary flow reserve was calculated as coronary sinus blood flow after dipyridamole infusion divided by the sinus blood flow at rest, and coronary resistance ratio (CRR) or coronary resistance reserve as resistance after dipyridamole infusion divided by resistance at baseline. Coronary venous and arterial oxygen saturation was measured using an Instrumentation Laboratory System 1302 blood gas analyser, in order to calculate oxygen content before and after infusion of dipyridamole. Myocardial oxygen consumption per $100 \mathrm{~g}$ left ventricular mass (MV02; ml. $\mathrm{min}^{-1} .100 \mathrm{~g}^{-1}$ ) was determined as the product of the coronary arterio-venous oxygen content difference and the normalized coronary sinus blood flow. Because contrast dye (ventriculography, coronary arteriography) may alter coronary dynamics ${ }^{[2]}$ the baseline value of coronary sinus blood flow was recorded not earlier than $20 \mathrm{~min}$ after any previous contrast dye injection.

\section{ENDOMYOCARDIAL BIOPSIES}

At the preoperative catheterization of the 18 patients studied postoperatively 2 to 3 left ventricular endomyocardial biopsies were obtained using the transseptal technique ${ }^{[28]}$. The samples were examined microscopically according to previously described techniques ${ }^{[28-30]}$. Muscle fibre diameter (MFD; $\mu$ ) was determined from several cross-sections at the level of the nucleus with a mechanical optical pen (MOP, Kontron GmbH, Zurich). The extent of non-muscular space (index of interstitial fibrosis, IF; \%) was evaluated with the point-counting method. Fibrous content $\left(\mathrm{FC} ; \mathrm{g} \cdot \mathrm{m}^{-2}\right.$ ) of the left ventricle was calculated

$$
\mathrm{FC}=\mathrm{LMMI} . \mathrm{IF} / 100
$$

where LMMI = left ventricular muscle mass index $\left(\mathrm{g} \cdot \mathrm{m}^{-2}\right)$; IF = relative interstitial fibrosis (\%).

\section{Statistics}

Values reported are means \pm standard deviations, unless otherwise indicated. Proportional distribution was tested by the chi-square-test, correlation by linear regression. Paired Student's $t$-test was used for comparing pre- and postoperative data in the patients who died after valve replacement. For multiple group comparison, one way analysis of variance was used, followed by a Newman-Keuls test for comparison between all pairs of means when an overall significant $P$ value $<0.05$ was obtained. To test baseline data and data after infusion of dipyridamole, a two-way analysis of variance with repeated measures was performed. If there was a significant 'group$x$ time' effect, both timepoints were examined separately, in order to explore the interaction. Differences between time points were then tested using the paired $t$-test.

\section{Results}

The three patient groups (preoperative, postoperative and controls) did not differ with respect to mean age $(58 \pm 10 ; 58 \pm 8 ; 50 \pm 10$ years) and mean body surface area $\left(1.82 \pm 0.13 ; 1.89 \pm 0.14 ; 1.83 \pm 0.11 \mathrm{~m}^{2}\right)$. No patients were anaemic and the mean haemoglobin contents were similar $\left(13.8 \pm \mathrm{l} \cdot 3 ; 14.4 \pm 0.8 ; 13.9 \pm 0.8{\mathrm{~g} . d l^{-1}}^{-1}\right.$.

The preoperative and postoperative patient groups comprised a similar distribution of patients with predominant aortic stenosis $(28 / 37$ vs $11 / 18$, ns) and aortic regurgitation $(9 / 37$ vs $7 / 18$, ns). Therefore data from these two patient groups were pooled for a first analysis of the results.

The postoperative patients had their diagnostic preoperative catheterization at our institution. Comparison of these preoperative data from postoperative patients with the preoperative patient group revealed a comparable severity of aortic valve disease in both groups. Patients of the preoperative and postoperative groups with predominant aortic stenosis showed a similar degree of reduction in the mean aortic valve area $\left(0.68 \pm 0.17\right.$ vs $\left.0.73 \pm 0.21 \mathrm{~cm}^{2} ; \mathrm{ns}\right)$ as well as concomitant aortic regurgitant fraction $(0.19 \pm 0.19$ vs $0.19 \pm 0 \cdot 15 ; \mathrm{ns})$. The preoperative patients with predominant aortic insufficiency had a slightly greater aortic regurgitant fraction than postoperative patients prior to aortic valve replacement $(0.61 \pm 0.08$ vs $0.48 \pm 0.06 ; P<0.01)$. No difference was found for left ventricular end-diastolic pressure, left ventricular systolic pressure, ejection fraction, left ventricular muscle mass index and wall thickness. Only left ventricular end-diastolic volume index was slightly smaller in the preoperative patient group than in the preoperative study of the postoperative patients $(126 \pm 37 \mathrm{vs}$ $\left.158 \pm 58 \mathrm{ml} . \mathrm{m}^{-2} ; P<0.05\right)$.

HAEMODYNAMIC AND ANGIOGRAPHIC DATA (TABLES IAND 2) Systolic aortic pressure was not different in the three groups (Table 1). Left ventricular end-diastolic pressure was higher in preoperative patients than controls (17 vs $9 \mathrm{mmHg} ; P<0.001$ ) and mean pulmonary capillary wed ge pressure was higher in preoperative than postoperative patients ( 10 vs $6 \mathrm{mmHg} ; P<0.01$ ). Left ventricular enddiastolic volume index was higher and ejection fraction was lower in preoperative compared with postoperative 
Table I Haemodynamic and angiographic dato

\begin{tabular}{|c|c|c|c|c|c|c|c|c|c|c|}
\hline & $\underset{(\mathrm{mmHg})}{\mathrm{SAP}}$ & $\underset{(\mathrm{mmHg})}{\text { LVSP }}$ & $\begin{array}{l}\text { LVEDP } \\
(\mathrm{mmHg})\end{array}$ & $\begin{array}{c}\mathrm{PCP} \\
(\mathrm{mmHg})\end{array}$ & $\begin{array}{c}\text { EDVI } \\
\left(\mathrm{ml} \mathrm{m}^{-2}\right)\end{array}$ & $\begin{array}{l}\text { LMMI } \\
\left(\mathrm{g} \mathrm{m}^{-2}\right)\end{array}$ & $\begin{array}{c}\mathrm{h} \\
(\mathrm{cm})\end{array}$ & $\begin{array}{l}\mathrm{EF} \\
(\%)\end{array}$ & $\begin{array}{c}\mathrm{S}_{\mathrm{cd}} \\
\text { (dyne. } 10^{3} \cdot \mathrm{cm}{ }^{2} \text { ) }\end{array}$ & $\frac{\mathrm{S}_{\mathrm{park}}}{\left(\text { dyne. } 10^{3} \cdot \mathrm{cm}^{2}\right)}$ \\
\hline $\begin{array}{l}\text { Preoperative patients } \\
(n=37)\end{array}$ & $130 \pm 19$ & $191 \pm 41$ & $17 \pm 8$ & $10 \pm 5$ & $126 \pm 37$ & $160 \pm 31$ & $1 \cdot 13 \pm 0 \cdot 14$ & $60 \pm 10$ & $52 \pm 28$ & $478 \pm 79$ \\
\hline $\begin{array}{l}\text { Postoperative patients } \\
(n=18)\end{array}$ & $131 \pm 16$ & - & - & $6 \cdot 4$ & $87 \pm 15$ & $99 \pm 17$ & $0.93 \pm 0.13$ & $67 \pm 8$ & $22 \pm 13$ & $354 \pm 71$ \\
\hline $\begin{array}{l}\text { Controls } \\
(n \square 7)\end{array}$ & $127 \pm 15$ & $122 \pm 27$ & $9 \pm 4$ & - & $112 \pm 40$ & $79 \pm 16$ & $0.71 \pm 0.08$ & $68 \pm 8$ & $41 \pm 18$ & $432 \pm 91$ \\
\hline \multicolumn{11}{|l|}{$P$ values } \\
\hline $\begin{array}{l}\text { Preoperative vs } \\
\text { postoperative }\end{array}$ & ns & - & - & $<0.01$ & $<0.001$ & $<0.001$ & $<0.001$ & $<0.05$ & $<0.01$ & $<0.001$ \\
\hline $\begin{array}{l}\text { Preoperative vs } \\
\text { controls }\end{array}$ & ns & $<0.01$ & $<0.001$ & - & ns & $<0.001$ & $<0.001$ & ns & $\mathrm{ns}$ & ns \\
\hline $\begin{array}{l}\text { Postoperative vs } \\
\text { controls }\end{array}$ & ns & - & - & - & ns & ns & $<0.01$ & ns & ns & $<0.05$ \\
\hline
\end{tabular}

Data are means $\pm S D . P$ values were obtained by one-way analysis of variance. $n s=$ not significant. $E D V I=$ left ventricular end-diastolic volume index; $E F=l e f t$ ventricular ejection fraction; $h=$ left ventricular end-diastolic wall thickness; $L M M I=$ left ventricular muscle mass index; $L$ VEDP = left ventricular end-diastolic pressure; $L V S P=$ left ventricular peak systolic pressure; $P C P=$ mean pulmonary capillary wedge pressure; $S A P=$ systolic aortic pressure; $S_{\text {ed }}=$ left ventricular circumferential end-diastolic wall stress; $S_{\text {pal }}=$ left ventricular circumferential peak systolic wall stress. 
Table 2 Haemodynamics and oxygen consumption at rest and after infusion of dipyridamole

\begin{tabular}{|c|c|c|c|c|c|c|c|c|c|c|c|c|}
\hline & \multicolumn{2}{|c|}{$\begin{array}{c}\text { HR } \\
\text { (beats.min-1) }\end{array}$} & \multicolumn{2}{|c|}{$\begin{array}{c}\mathrm{MAP} \\
(\mathrm{mmHg})\end{array}$} & \multicolumn{2}{|c|}{$\underset{(\mathrm{mmHg})}{\mathrm{CSP}}$} & \multicolumn{2}{|c|}{$\begin{array}{c}\text { MAP-CSP } \\
(\mathrm{mmHg})\end{array}$} & \multicolumn{2}{|c|}{$\begin{array}{c}\text { A-VDO, } \\
(\%)\end{array}$} & \multicolumn{2}{|c|}{$\begin{array}{c}\mathrm{MVO}_{2} \\
\left(\mathrm{ml} \cdot \mathrm{min}^{-1} \cdot 100 \mathrm{~g}^{-1}\right)\end{array}$} \\
\hline & $\mathbf{R}$ & D & $\mathbf{R}$ & D & $\mathbf{R}$ & D & $\mathbf{R}$ & D & $\mathbf{R}$ & D & $\mathrm{R}$ & D \\
\hline \multirow{2}{*}{$\begin{array}{l}\text { Preoperative patients } \\
(\mathrm{n}=37) \\
P \text { values } \mathrm{R} \text { vs } \mathrm{D}:\end{array}$} & $73 \pm 11$ & $87 \pm 14$ & $92 \pm 11^{*}$ & $88 \pm 12^{*}$ & $4 \pm 2$ & $5 \pm 2$ & $87 \pm 11^{*}$ & $83 \pm 11^{*}$ & $66 \pm 4 \dagger$ & $43 \pm 10^{*} \ddagger$ & $11 \pm 6$ & $11 \pm 5$ \\
\hline & \multicolumn{2}{|c|}{$<0.001$} & \multicolumn{2}{|c|}{$<001$} & \multicolumn{2}{|c|}{ ns } & \multicolumn{2}{|c|}{$<0.01$} & \multicolumn{2}{|c|}{$<0.001$} & \multicolumn{2}{|c|}{ ns } \\
\hline \multirow{2}{*}{$\begin{array}{l}\text { Postoperative patients } \\
(\mathrm{n}=18) \\
P \text { values } \mathrm{R} \text { vs } \mathrm{D}:\end{array}$} & $74 \pm 16$ & $85 \pm 15$ & $100 \pm 11$ & $96 \pm 9$ & $3 \pm 2$ & $3 \pm 2$ & $97 \pm 12$ & $92 \pm 10$ & $64 \pm 6$ & $36 \pm 12^{* *}$ & $12 \pm 5$ & $13 \pm 5$ \\
\hline & \multicolumn{2}{|c|}{$<0.001$} & \multicolumn{2}{|c|}{$<0.01$} & \multicolumn{2}{|c|}{ ns } & \multicolumn{2}{|c|}{$<0.01$} & \multicolumn{2}{|c|}{$<0.001$} & \multicolumn{2}{|c|}{ ns } \\
\hline \multirow{2}{*}{$\begin{array}{l}\text { Controls } \\
(n=7) \\
P \text { values } R \text { vs } D:\end{array}$} & $67 \pm 12$ & $87 \pm 16$ & $95 \pm 9$ & $91 \pm 6$ & $4 \pm 1$ & $5 \pm 2$ & $91 \pm 9$ & $87 \pm 6$ & $61 \pm 5$ & $21 \pm 5$ & $14 \pm 3$ & $13 \pm 5$ \\
\hline & \multicolumn{2}{|c|}{$<0-001$} & \multicolumn{2}{|c|}{ ns } & \multicolumn{2}{|c|}{ n8 } & \multicolumn{2}{|c|}{ ns } & \multicolumn{2}{|c|}{$<0.001$} & \multicolumn{2}{|c|}{ ns } \\
\hline
\end{tabular}

Data are means $\pm S D$. P values were obtained by paired $t$-test. $n s=$ not signficant. A-VDO $=$ coronary arterio-venous difference of oxygen saturation; CSP $m$ mean coronary sinus pressure; $D=$ after

dipyridamole infusion; $\mathrm{HR}$ = heart rate; $\mathrm{MAP}=$ mean aortic pressure; $M V \mathrm{O}_{2}=$ myocardial oxygen consumption per minute and per $100 \mathrm{~g}$ of left ventricular muscle mass; $\mathrm{R}=$ rest.

$*=P<0.05$ preoperative vs postoperative; $t=P<0.05$ preoperative vs controls; $\ddagger=P<0.001$ preoperative vs controls; ** $=P<0.01$ postoperative vs controls (ANOVA). 

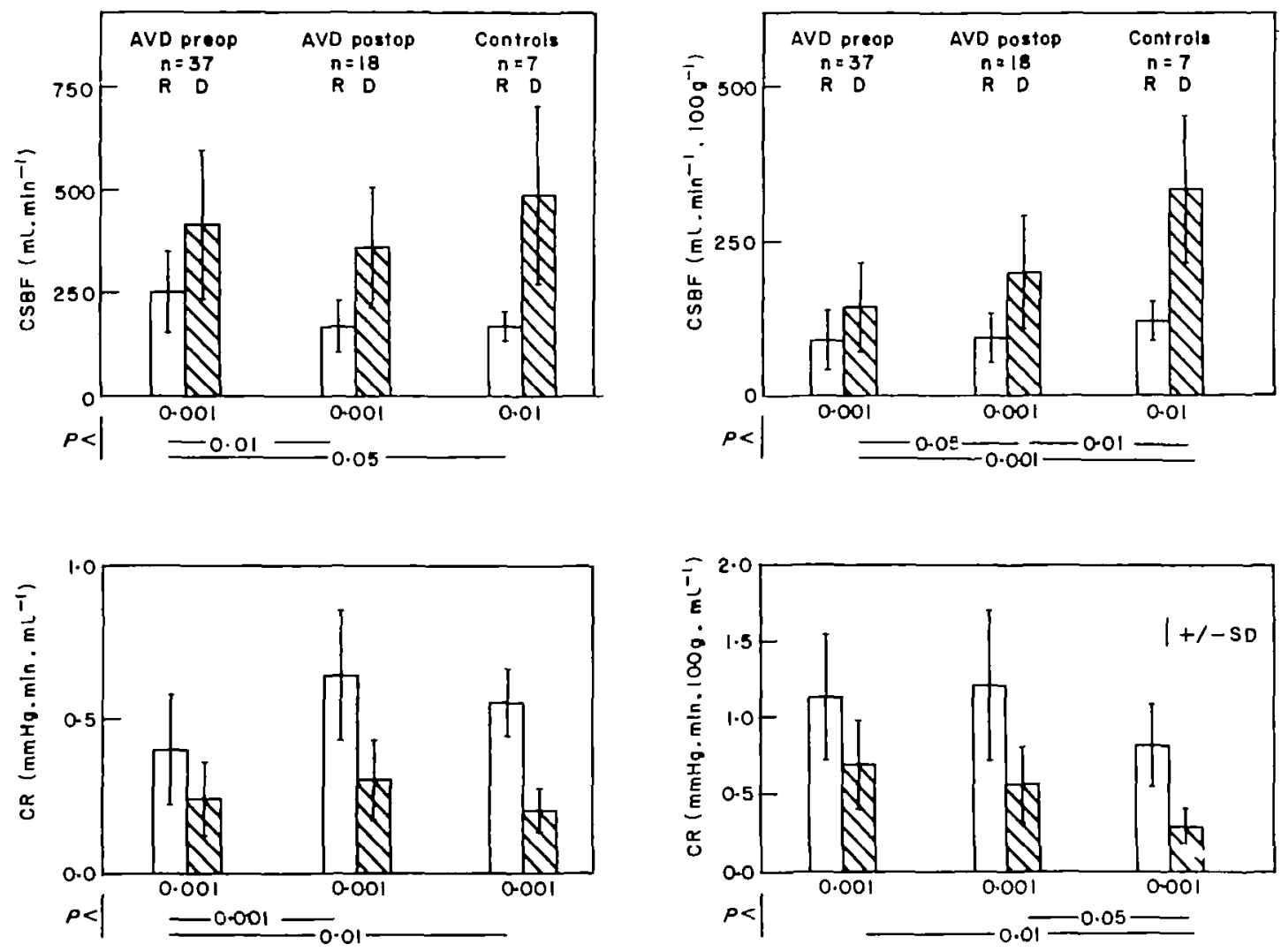

Figure I Coronary sinus blood flow (upper panels) and coronary resistance (lower panels) before and after dipyridamole infusion. Resting (R) total coronary sinus blood flow (CSBF; ml.min ${ }^{-1}$ ) was higher and total coronary resistance (CR; $\mathrm{mmHg} \cdot \mathrm{min} \cdot \mathrm{ml}^{-1}$ ) (left panels) significantly lower in preoperative patients with aortic valve (AVD preop) disease compared with postoperative patients (AVD postop) and with controls, whereas achieved maximal flow and resistance after dipyridamole infusion (D) did not differ. Conversely, resting coronary sinus blood flow normalized per $100 \mathrm{~g}$ left ventricular muscle mass (CSBF; ml.min ${ }^{-1} .100 \mathrm{~g}^{-1}$ ) and resistance normalized per $100 \mathrm{~g}$ left ventricular muscle mass (CR: $\mathrm{mmHg} \cdot \mathrm{min} 100 \mathrm{~g} \cdot \mathrm{ml}^{-1}$ ) (right panels) were similar in the three groups. Achieved maximal flow per $100 \mathrm{~g}$ left ventricular mass was reduced in preoperative patients and was restored at least in part in postoperative patients.

patients but differences from controls did not reach statistical significance. Left ventricular muscle mass index and wall thickness were significantly greater in the preoperative patient group than in postoperative patients and in controls. Between the two latter groups there was a significant difference in wall thickness $(0.93$ vs $0.71 \mathrm{~cm}$; $P<0.01)$. Left ventricular circumferential end-diastolic and peak systolic stress were higher in preoperative than postoperative patients.

After infusion of dipyridamole, heart rate increased in all three groups (average between 11 and 20 beats $\min ^{-1}$ ) and mean aortic pressure fell (average $4 \mathrm{mmHg}$ ) (Table 2). There were no differences in myocardial oxygen consumption per $100 \mathrm{~g}$ left ventricular muscle mass among the three patient groups, neither at rest nor after dipyridamole.

\section{CORONARY DYNAMICS (FIGURES I-3, TABLE 3)}

At rest coronary sinus blood flow was significantly higher in preoperative than in postoperative patients and controls $(252 \pm 99$ vs $169 \pm 63, P<0.01$; vs $170 \pm 35$ ml.min ${ }^{-1}, P<0.05$ ) (Fig. 1). Maximally achieved flow, however, did not differ significantly ( $416 \pm 184$ vs $361 \pm 150$ vs $488 \pm 235 \mathrm{ml} \cdot \mathrm{min}^{-1}$ ). Corresponding coronary resistance was lower in preoperative than in postoperative patients and controls $(0.40 \pm 0.18$ vs $0.64 \pm 0.21, P<0.001$; vs $0.55 \pm 0.11 \mathrm{mmHg} \cdot \mathrm{min} \cdot \mathrm{ml}^{-1}, P<0.01$ ), while minimal coronary resistance after administration of dipyridamole showed no significant differences $(0.24 \pm 0.12$ vs $0.30 \pm$ 0.13 vs $\left.0.20 \pm 0.07 \mathrm{mmHg} \cdot \mathrm{min} \cdot \mathrm{ml}^{-1}\right)$. Conversely, coronary flow per $100 \mathrm{~g}$ left ventricular muscle mass was similar at rest within the three groups ( $90 \pm 48$ vs $94 \pm 40$ vs $121 \pm 32 \mathrm{ml} \cdot \mathrm{min}^{-1} \cdot 100 \mathrm{~g}^{-1}$ ), but significantly lower after infusion of dipyridamole in the preoperative patient group compared with postoperative patients and controls $(144 \pm 72$ vs $200 \pm 92, P<0.05$; vs $336 \pm 130$ ml.min $\left.\min ^{-1} .100 \mathrm{~g}^{-1}, P<0.001\right)$ and also between the two latter groups $(P<0.01)$. Similarly, at rest no differences were found among the groups for coronary resistance, when it was normalized to $100 \mathrm{~g}$ left ventricular muscle mass $(1.13 \pm 0.41$ vs $1.21 \pm 0.49$ vs $0.81 \pm 0.27$ $\mathrm{mmHg} \cdot \mathrm{min} \cdot 100 \mathrm{~g} \cdot \mathrm{ml}^{-1}$ ). However, after dipyridamole, resistance was higher in preoperative and postoperative patients compared with controls $(0.69 \pm 0.29$ vs $0.56 \pm$ 

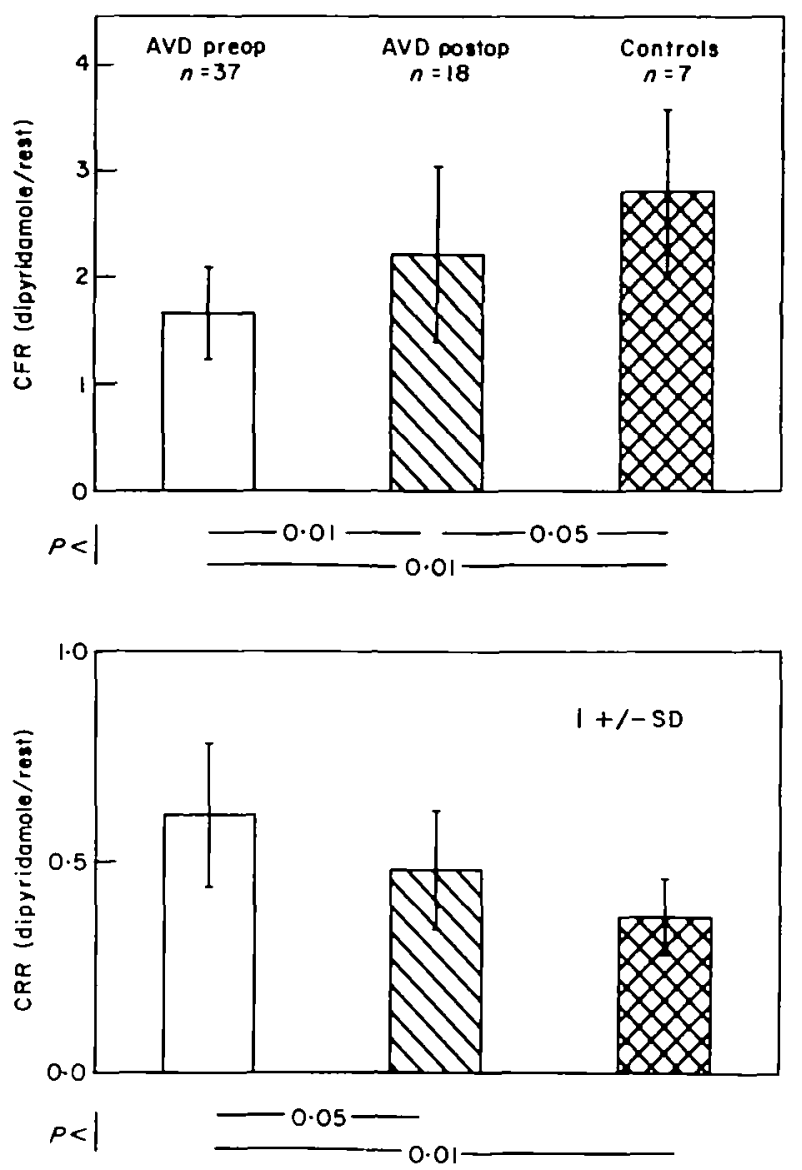

Figure 2 Coronary flow reserve (upper panel) and coronary vasodilator capacity (lower panel). Patients after aortic valve replacement (AVD postop) showed a distinct improvement of coronary flow reserve, assessed from coronary flow ratio (CFR), compared with preoperatıve patients with aortic valve disease (AVD preop) Similarly coronary vasodilator capacity, assessed from coronary resistance ratio (CRR), was improved.

$0 \cdot 25$, ns; vs $0.29 \pm 0.12 \mathrm{mmHg} \cdot \mathrm{min} .100 \mathrm{~g} \cdot \mathrm{ml}^{-1}, P<0.01$, $P<0.05$ respectively).

Coronary flow ratio was significantly lower in preoperative patients than in postoperative patients and controls $(1.66 \pm 0.44$ vs $2.22 \pm 0.85, P<0.01$; vs $2.80 \pm$ $0.84, P<0.01)$ and lower in postoperative patients than controls $(p<0.05)$ (Fig. 2). Corresponding coronary resistance ratio was higher in preoperative than in postoperative patients or controls $(0.61 \pm 0.17$ vs $0.48 \pm 0.14$, $P<0.05$; vs $0.37 \pm 0.10, P<0.01$ ). Differences between postoperative patients and controls did not reach statistical significance. The improvement of coronary flow and resistance ratio was concomitant with the regression of left ventricular hypertrophy (Fig. 3), but a significant linear correlation between the two parameters could not be established. Furthermore no correlation could be shown between coronary flow or resistance ratio and left ventricular systolic pressure, left ventricular end-diastolic pressure, pulmonary capillary wedge pressure, systolic, diastolic and mean aortic pressure or ejection fraction for each group separately or for pooled data.
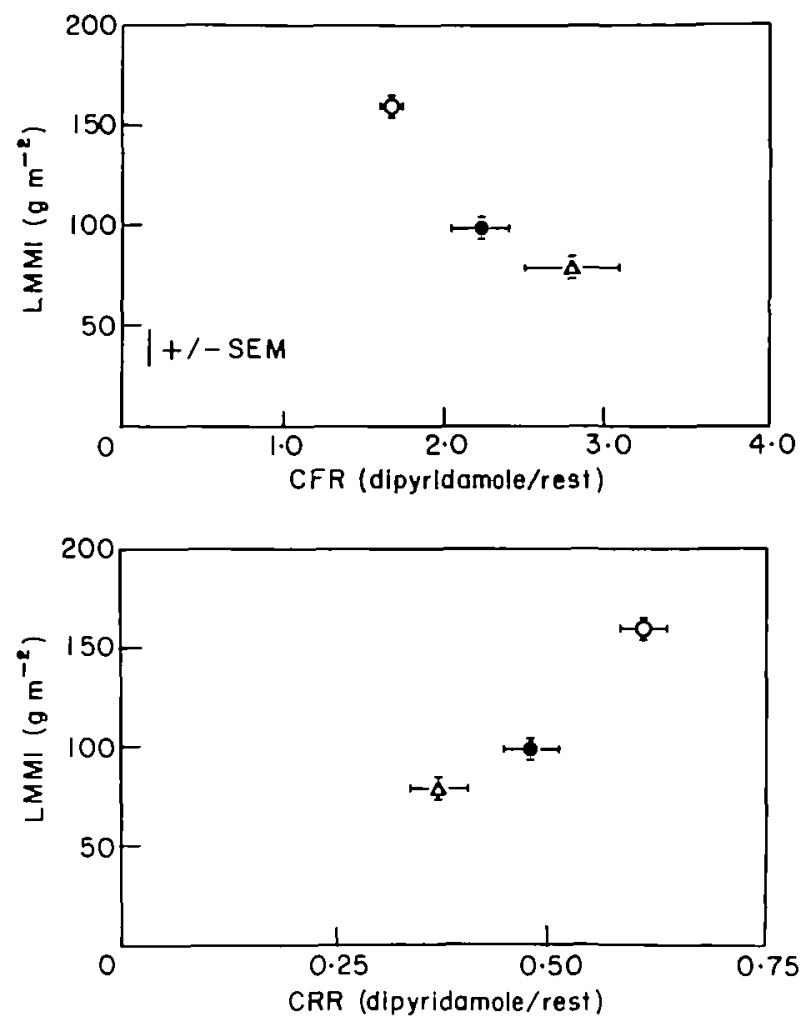

Figure 3 Relationship between coronary flow reserve (upper panel) and coronary resistance ratio (lower panel) to left ventricular muscle mass index. Patients $\mathbf{3 0}$ months after aortic valve replacement (•) showed a distinct regression of left ventricular muscle mass index (LMMl; g. $\mathrm{m}^{-2}$ ) compared with preoperative patients $(O)(P<0.01)$ and no significant difference from controls $(\Delta)$. Concomitant with the regression of muscle mass was the improvement of coronary flow reserve (CFR) and coronary resistance ratio (CRR) in postoperative compared with preoperative patients $(P<0.05)$.

Nine of the 18 postoperative patients showed a normal flow ratio $(\geq 2 \cdot 2$; group $A)$ and nine an abnormal flow ratio ( $<2 \cdot 2$; group B) (Table 3 ). These two subgroups showed no differences with respect to preoperative left ventricular hypertrophy (left ventricular muscle mass index, wall thickness) and morphometric measurements. The Doppler echocardiographically determined postoperative residual pressure gradient over the aortic prosthesis was similar within these two subgroups $(18.6 \pm 4 \cdot 9$ vs $19 \cdot 5 \pm 9 \cdot 8, \mathrm{~ns} ; \mathrm{n}=8$ in each group). No correlations existed between coronary flow or resistance ratio and preoperative ejection fraction, left ventricular end-diastolic pressure, left ventricular systolic pressure, number of months after aortic valve replacement, postoperative residual pressure gradient and extent of regression of left ventricular muscle mass, the latter being evaluated as ratio of left ventricular muscle mass index before and after surgery.

PATIENTS WITH IDENTICAL PERFUSION PRESSURES (TABLE 4)

During maximal vasodilatation, the pressure-flow relationship is steep, and small changes in perfusion pressure produce large changes in coronary flow and 
Table 3 Morphometric measurements before aortic valve replacement

\begin{tabular}{llcccc}
\hline \multirow{2}{*}{ Patient } & $\begin{array}{c}\text { LMMI } \\
\left(\mathrm{g} \cdot \mathrm{m}^{-2}\right)\end{array}$ & $\begin{array}{c}\mathrm{h} \\
(\mathrm{cm})\end{array}$ & $\begin{array}{c}\text { MFD } \\
(\mu)\end{array}$ & $\begin{array}{c}\text { IF } \\
(\%)\end{array}$ & $\begin{array}{c}\text { FC } \\
\left(\mathrm{g} \mathrm{m}^{-2}\right)\end{array}$ \\
\hline
\end{tabular}

Group A: Normal flow reserve after AVR

$\begin{array}{lrrrrrc} & 1 & 117 & 0.89 & 28.2 & 10 & 11.7 \\ & 2 & 183 & 1.38 & 31.4 & 16 & 29.3 \\ & 3 & 194 & 1.21 & 30.0 & 16 & 30.5 \\ & 4 & 159 & 1.19 & 29.1 & 13 & 20.7 \\ & 5 & 266 & 1.14 & 26.7 & - & - \\ & 6 & 236 & 1.25 & 30.3 & 27 & 63.7 \\ & 7 & 169 & 0.86 & 34.6 & 18 & 30.4 \\ & 8 & 164 & 1.02 & 35.9 & 21 & 33.8 \\ & 9 & 135 & 1.03 & 30.3 & 14 & 19.0 \\ \text { Mean } & & 180 & 1.11 & 30.7 & 17 & 29.9 \\ \text { SD } & & 47 & 0.17 & 2.9 & 5 & 15.6 \\ & & & & & & \end{array}$

Group B: Abnormal flow reserve after AVR

\begin{tabular}{lrrrrrr} 
& 1 & 116 & 0.98 & $32 \cdot 7$ & 8 & $9 \cdot 3$ \\
& 2 & 132 & 0.92 & $35 \cdot 9$ & 12 & $15 \cdot 8$ \\
& 3 & 147 & 0.81 & $32 \cdot 1$ & 15 & $22 \cdot 1$ \\
& 4 & 175 & $1 \cdot 25$ & $38 \cdot 6$ & 29 & $50 \cdot 1$ \\
& 5 & 143 & 1.04 & $31 \cdot 7$ & 12 & $17 \cdot 7$ \\
& 6 & 122 & 0.82 & $32 \cdot 6$ & 22 & $26 \cdot 8$ \\
& 7 & 187 & 0.98 & $29 \cdot 7$ & 13 & $24 \cdot 3$ \\
& 8 & 200 & $1 \cdot 16$ & $32 \cdot 2$ & 13 & $26 \cdot 0$ \\
& 9 & 116 & 0.98 & $35 \cdot 3$ & 15 & $17 \cdot 4$ \\
Mean & & 149 & 0.99 & $33 \cdot 4$ & 15 & $23 \cdot 3$ \\
SD & & 32 & $0 \cdot 14$ & $2 \cdot 7$ & 6 & $11 \cdot 5$ \\
\hline
\end{tabular}

There were no significant differences between the two groups. $A V R=$ aortic valve replacement; $F C=$ left ventricular fibrous content; IF = relative interstitial fibrosis; MFD a muscle fibre diameter. Endomyocardial biopsy of patient 5 , group A, was not suitable for quantitatıve analysis of relative interstitial fibrosis.

thus flow reserve ${ }^{[19}$. Perfusion pressures (mean aortic pressure - mean coronary sinus pressure) after vasodilatation with dipyridamole differed significantly between preoperative and postoperative patients (average difference of $9 \mathrm{mmHg} ; P<0.05$ ) (Table 2). This could have contributed to our findings. We therefore performed a second analysis of the data including only patients having perfusion pressures after dipyridamole equal to the controls \pm 2 SD (Table 4). The same overall result was found in this subset of patients. In the preoperative patients coronary sinus blood flow at rest was higher and coronary resistance was lower $(P<0.05)$, than in the postoperative patients, but there was no statistically significant difference from controls. Coronary flow and resistance after infusion of dipyridamole were similar in the three groups. Coronary flow reserve was lower in preoperative than in postoperative patients $(P<0.05)$ or controls $(P<0.01)$. Conversely, coronary resistance ratio was higher in the preoperative than the postoperative group $(P<0.05)$ or controls $(P<0.01)$. Differences between postoperative patients and controls did not reach statistical significance. The extent of left ventricular hypertrophy in preoperative patients and regression of hypertrophy in postoperative patients of this subgroup were nearly identical to the values reported for the whole patient population (Tables 1 and 4).

AORTC STENOSIS VS AORTIC INSUFFICIENCY (FIG. 4)

Coronary flow and resistance ratios of patients with predominant aortic stenosis and with predominant aortic insufficiency were nearly identical preoperatively (1.62 士 0.40 vs $1.76 \pm 0.51$, ns; $0.62 \pm 0.17$ vs $0.59 \pm 0.15$, ns) as well as postoperatively $(2 \cdot 27 \pm 0.87$ vs $2 \cdot 15 \pm 0.87$, ns vs $0.47 \pm 0.15$ vs $0.49 \pm 0.15$, ns) (Fig. 4). Coronary flow ratio in preoperative patients with predominant aortic stenosis $(\mathrm{n}=28)$ was lower than in postoperative patients $(\mathrm{n}=11)$ and in controls $(\mathrm{n}=7)(1.62 \pm 0.40$ vs $2.27 \pm 0.87, P<0.01$; vs $2.80 \pm 0.84, P<0.01)$ and, conversely, coronary resistance ratio was higher in preoperative patients with aortic stenosis than in postoperative patients and controls $(0.62 \pm 0.17$ vs $0.47 \pm 0.15, \quad P<0.05 ;$ vs $0.37 \pm 0.10$, $P<0.01)$. Differences of coronary flow and resistance reserve between postoperative patients with aortic stenosis and controls did not reach statistical significance. In preoperative patients with predominant aortic insufficiency $(n=9)$ coronary flow ratio was significantly lower $(1 \cdot 76 \pm 0.51$ vs $2.80 \pm 0.84, P<0.05)$ and coronary resistance ratio was significantly higher than in controls $(0.59 \pm 0.15$ vs $0.37 \pm 0.10, P<0.05)$. In postoperative patients with predominant aortic insufficiency $(n=7)$ coronary flow ratio tended to be higher than in preoperative patients with aortic insufficiency and lower than in controls $(1 \cdot 76 \pm 0.51$ vs $2 \cdot 15 \pm 0 \cdot 87$, ns; vs $2 \cdot 80 \pm 0 \cdot 84$, ns), and conversely, coronary resistance ratio in postoperative patients with predominant aortic insufficiency tended to be lower than in preoperative patients and higher than in controls $(0.59 \pm 0.15$ vs $0.49 \pm 0.15$, ns; vs $0.37 \pm 0 \cdot 10$, ns), but differences did not reach statistical significance. The degree of aortic regurgitation was somewhat higher in preoperative patients than in postoperative patients, however, no relationship could be established between severity of aortic regurgitation and coronary flow or resistance ratio preoperatively and postoperatively.

Left ventricular hypertrophy, as assessed by left ventricular muscle mass index, was similar in preoperative patients with predominant aortic stenosis and predominant aortic insufficiency $\left(155 \pm 26\right.$ vs $175 \pm 40$ g.m ${ }^{-2}$, ns), as was extent of regression of hypertrophy in postoperative patients with predominant aortic stenosis and aortic insufficiency $\left(101 \pm 17\right.$ vs $98 \pm 19$ g.m $\mathrm{m}^{-2}$, ns $)$.

\section{Discussion}

Chronic pressure or volume overload resulting in left ventricular hypertrophy is associated with a decreased coronary vasodilator capacity ${ }^{[2-8]}$. In patients with aortic stenosis ${ }^{[0]}$ and insufficiency ${ }^{[10]}$ and normal coronary arteries the decreased coronary vasodilator reserve is thought to be the underlying cause of ischaemia and anginal symptoms because it interferes with an adequate increase of coronary blood flow under stress. In animal experiments with chronic overload states increased muscle mass per se has been determined to be the main 
Table 4 Subgroup of patients with identical perfusion pressures after infusion of dipyridamole

\begin{tabular}{|c|c|c|c|c|c|c|c|c|c|}
\hline & \multicolumn{2}{|c|}{$\begin{array}{l}\text { MAP-CSP } \\
(\mathrm{mmHg})\end{array}$} & \multicolumn{2}{|c|}{$\begin{array}{c}\text { CSBF } \\
\left(\mathrm{ml} \cdot \mathrm{min}^{-1}\right)\end{array}$} & \multicolumn{2}{|c|}{$\underset{\left(\operatorname{mmHg} \cdot \min \cdot \mathrm{ml}^{-1}\right)}{\mathrm{CR}}$} & \multirow[t]{2}{*}{ CFR } & \multirow[t]{2}{*}{ CRR } & \multirow{2}{*}{$\begin{array}{l}\text { LMMI } \\
\left(\mathrm{g} \mathrm{m}^{-7}\right)\end{array}$} \\
\hline & $\mathbf{R}$ & D & $\mathbf{R}$ & D & $\mathbf{R}$ & D & & & \\
\hline $\begin{array}{l}\text { Preoperative } \\
\text { patients }(\mathrm{n}=26)\end{array}$ & $89 \pm 8$ & $86 \pm 7$ & $248 \pm 113$ & $431 \pm 203 \dagger$ & $0.43 \pm 0.20$ & $0.25 \pm 0.14 \dagger$ & $1.75 \pm 0.46$ & $0.60 \pm 0 \cdot 18$ & $153 \pm 28$ \\
\hline $\begin{array}{l}\text { Postoperative } \\
\text { patients }(n=14)\end{array}$ & $92 \pm 9$ & $88 \pm 6$ & $169 \pm 68$ & $367 \pm 164 \dagger$ & $0.61 \pm 0 \cdot 18$ & $0 \cdot 29 \pm 0 \cdot 13^{*}$ & $2 \cdot 27 \pm 0.95$ & $0.48 \pm 0.16$ & $100 \pm 18$ \\
\hline $\begin{array}{l}\text { Controls } \\
(n=7)\end{array}$ & $91 \pm 9$ & $87 \pm 6$ & $170 \pm 35$ & $488 \pm 235^{*}$ & $0.55 \pm 0 \cdot 11$ & $0.20 \pm 0.07 \dagger$ & $2 \cdot 80 \pm 0 \cdot 84$ & $0 \cdot 37 \pm 0 \cdot 10$ & $79 \pm 16$ \\
\hline \multicolumn{10}{|l|}{$P$ values } \\
\hline $\begin{array}{l}\text { Preoperative vs } \\
\text { postoperative }\end{array}$ & ns & ns & $<0.05$ & ns & $<0.05$ & ns & $<0.05$ & $<0.05$ & $<0-001$ \\
\hline $\begin{array}{l}\text { Preoperative vs } \\
\text { controls }\end{array}$ & ns & ns & ns & ns & ns & ns & $<0.01$ & $<0.01$ & $<0.001$ \\
\hline $\begin{array}{l}\text { Postoperative vs } \\
\text { controls }\end{array}$ & ns & ns & ns & ns & ns & ns & $\mathrm{ns}$ & ns & ns \\
\hline
\end{tabular}

Data are means $\pm S D$. $P$ values were obtained by one-way analysis of variance. $n s=$ not significant. $C F R=$ coronary flow reserve; $C R=$ coronary resistance; $C R R=$ coronary resistance ratio; $C S B F=$ coronary sinus blood flow; $C S P=$ mean coronary sinus pressure; $D=$ after dipyridamole infusion; $\mathrm{LMMI}=$ left ventricular muscle mass index; $M A P=$ mean aortic pressure; $\mathrm{R}=$ rest. $* P<0.01 ; \dagger=P<0.001(\mathrm{R}$ vs $\mathrm{D}$; paired $t$-test).

factor responsible for the derangements of flow and resistance reserve $e^{[14,31,32]}$. Wicker et al. ${ }^{[17}$ have shown that in rats with left ventricular hypertrophy a normal coronary flow reserve is restored with reversal of hypertrophy. Regression of left ventricular hypertrophy in man after successful aortic valve replacement is well known ${ }^{[18,19]}$. In order to establish whether, in concert with this regression, an improvement of coronary reserve occurs, we compared coronary flow and resistance reserve in 18 patients after aortic valve replacement whose angiographic mass had decreased from $165 \pm 42$ to $99 \pm 17 \mathrm{~g} \cdot \mathrm{m}^{-2}$ postoperatively with that of 37 preoperative patients with aortic valve disease.

\section{CORONARY FLOW AND RESISTANCE RESERVE}

Coronary flow ratio (dipyridamole/rest) was lower in preoperative than in postoperative patients or controls, whereas corresponding coronary resistance ratio (dipyridamole/rest) was higher in preoperative patients than in the other two patient groups (Fig. 2). Differences in the flow but not in the resistance ratio between patients after aortic valve replacement and controls were significant. To minimize the influence of perfusion pressure on coronary flow ratio $^{[19]}$, patients with identical perfusion pressures after infusion of dipyridamole were examined separately and a similar result was found. Comparison of patients with predominant aortic stenosis and patients with predominant aortic insufficiency showed a similar reduction of coronary flow reserve preoperatively and a similar enhancement postoperatively (Fig. 4). Due to the small number of patients with predominant aortic insufficiency studied postoperatively, the extent of this enhancement is more difficult to judge conclusively in this particular subgroup.

These data confirm the observations of others ${ }^{[1,2,5,8-10]}$ who found a decreased coronary flow reserve in patients with aortic valve disease. In addition they reveal a major new finding that, indeed, coronary flow reserve is distinctly improved after aortic valve replacement. This improvement accompanied the expected regression of left ventricular hypertrophy 30 months after aortic valve replacement. Regression of left ventricular hypertrophy was not complete and correspondingly coronary flow reserve was not totally normalized (Fig. 3). However, we and others ${ }^{[7]}$ did not find a linear correlation between left ventricular muscle mass and coronary flow reserve. In experimental aortic valve disease Su-Fan et al. ${ }^{[32]}$ have described an inverse and logarithmic relationship.

\section{POSSIBLE MECHANISMS OF IMPAIRED CORONARY VASODILATOR CAPACITY}

The comparison of preoperative and postoperative basal coronary flow and flow after maximal vasodilation with dipyridamole provides important information as to possible mechanisms responsible for the decreased coronary vasodilator capacity in hypertrophy. In preoperative patients with aortic valve disease we found a distinct increase in resting coronary flow and a lower coronary resistance than in controls or postoperative patients (Fig. 1), whereas achieved maximal flow and minimal coronary resistance were similar in all three patient groups. This observation is in agreement with previous studies in animals ${ }^{(13,17,31-34]}$ and in man ${ }^{[1,3,109}$ which found elevated basal coronary flow and lower resistance in hypertrophied 

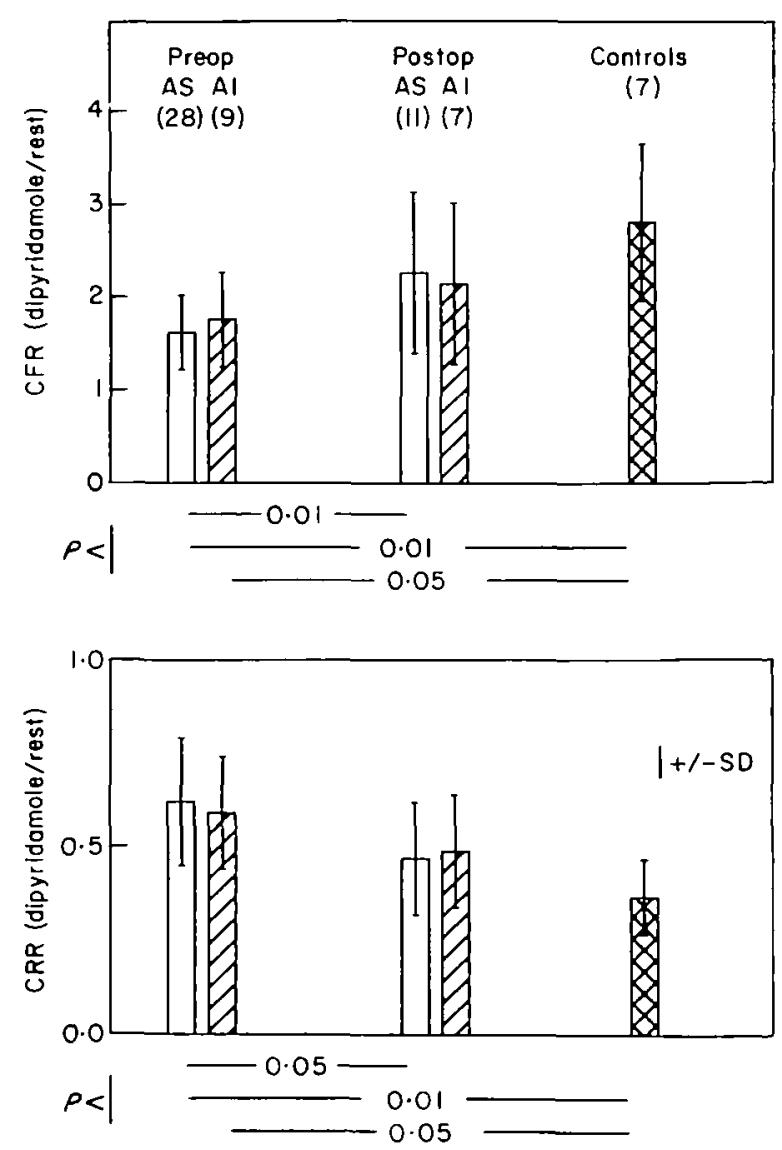

Figure 4 Comparison between patients with aortic stenosis and patients with aortic insufficiency. Coronary flow reserve (CFR) (upper panel) and coronary resistance reserve (CRR) (lower panel) were similar preoperatively and had changed similarly in postoperative patients. ( ) number of patients in each group.

hearts. It is also in agreement with the data of Kawachi et $a l .{ }^{[35]}$, who described a significant decrease of basal coronary flow in patients with aortic insufficiency 7 months after aortic valve replacement. This finding of an increased preoperative basal flow implies that a large portion of coronary reserve is used at rest to cover the demands caused by the hypertrophied myocardium. It further implies that the increase in left ventricular muscle mass is not paralleled by an appropriate change of cross-sectional area of the vascular bed ${ }^{(13,16,32,33,39}$.

The role of a massively altered extravascular component (i.e. compressive forces) in reducing coronary flow reserve is not entirely clear. In isolated, adenosinevasodilated, blood perfused canine cardiac preparations, Harrison et al.$^{[37}$ found similar transmural and collateral coronary resistance per $100 \mathrm{~g}$ of normal and hypertrophied myocardium. In these preparations extravascular compressive forces were minimized and equalized between normal and hypertrophied hearts. However, others ${ }^{[13,38]}$ have demonstrated an increased minimal vascular resistance in the hypertrophied myocardium which may be due in part to extravascular compressive forces. In patients with supravalvular aortic stenosis and left ventricular hypertrophy no improvement of coronary vasodilator capacity occurred when only the intramural compressive forces were normalized by operation ${ }^{[39]}$. In our postoperative patients diastolic (mean pulmonary capillary wedge pressure, end-diastolic wall stress) and systolic compressive forces (systolic aortic pressure, peak systolic wall stress) were lowest among the three groups. It is recognized however that both peak systolic and end-diastolic circumferential wall stress were somewhat underestimated in the postoperative patients because systolic aortic instead of systolic left ventricular pressure and mean pulmonary capillary wedge instead of left ventricular end-diastolic pressure were used for stress calculations. Nevertheless, one would have expected an increase in maximal achievable coronary flow after aortic valve replacement if the preoperative reduction in coronary vasodilator capacity were due largely to systolic and diastolic compressive forces. However, in the postoperative group, total maximal vasodilated flow was even slightly, although not significantly, smaller than in the two other groups (Fig. 1). Thus compressive forces are unlikely to have played a role in the preoperative reduction of coronary flow and vasodilator reserve.

The slightly lower maximal flow after dipyridamole infusion in the postoperative group compared with the other two groups raises the question of whether, in aortic valve disease, the longstanding hypertrophy leads to a structural alteration of the coronary vascular bed. An increase in left ventricular fibrous content has been reported in aortic valve disease ${ }^{[28.300]}$. In the present study there was, however, no correlation between preoperative nonmuscular interstitial space assessed as relative interstitial fibrosis or total left ventricular fibrous content and the postoperative coronary flow reserve (Table 3). Although in left ventricular hypertrophy some enlargement of epicardial coronary vessels has been demonstrated ${ }^{16,401}$, no consistent morphological abnormalities in coronary resistance vessels, even in hypertrophy due to arterial hypertension, could be established ${ }^{[7.40]}$. Thus, it is unlikely that the observed preoperative increase of interstitial fibrous tissue in aortic valve disease was accompanied by a significant reduction of the cross-sectional area of the coronary vascular bed either from rarefaction or wall thickening and luminal narrowing of the resistance vessels.

\section{LIMITATIONS OF THE STUDY}

Coronary sinus thermodilution techniques ${ }^{[24]}$ were used to assess total coronary sinus blood flow. This method is unable to measure the flow in specific ventricular layers or regions $\mathrm{s}^{[1]}$ and may be inaccurate after interventions leading to coronary sinus reflux ${ }^{[25]}$. In the absence of coronary artery disease and no known movement of the catheter, the thermodilution technique is adequate for measuring relatively slow and large changes of coronary blood flow $w^{[4]}$, as observed in our study.

For the measurement of maximal vasodilator capacity dipyridamole was used ${ }^{[15,27]}$. It is recognized that dipyridamole at the chosen dose $\left(0.5 \mathrm{mg} \mathrm{kg}{ }^{-1}\right)$ does not always produce maximal coronary dilation ${ }^{[42]}$. Nevertheless, 
maximal achieved flows at identical perfusion pressures were similar in the three patient groups (Table 4), suggesting a similar effect of dipyridamole in all groups. The duration of dipyridamole infusion was $15 \mathrm{~min}$ to minimize the systemic effects on heart rate and blood pressure ${ }^{[2,26]}$. A fall in blood pressure would have been especially untoward in the patients with aortic stenosis. It is speculated that the prolonged administration of dipyridamole was responsible for the generally low coronary flow ratios in the present study. The average value in the control group was 2.8 with a range of $2 \cdot 2$ to $4 \cdot 3$. These values, considered normal, are somewhat low, but similar low ratios have been reported by others, who administered dipyridamole 0.5 and $0.75 \mathrm{mg} . \mathrm{kg}^{-1}$ i.v. within 4-6 $\mathrm{min}^{[7,10,42-44]}$. In particular, Rossen et al..$^{[4]}$ found a coronary flow reserve in the same range in 8 of 12 normal subjects in whom coronary vasospasms were excluded by an ergonovine test.

It is assumed that the control subjects had a normal coronary reserve, although in patients with ischaemialike symptoms and normal coronary arteries a reduced coronary flow reserve has been reported ${ }^{[4,45]}$. Patients with true microvascular angina who also had a documented abnormal vasodilator capacity during dynamic exercise showed clearly smaller flow ratios than the present control subjects ${ }^{[46]}$. Moreover, maximal vasodilated flow of our control group was similar to previously published data on coronary blood flow after i.v. dipyridamole in normal subjects ${ }^{[10,27,43,44]}$.

\section{Conclusions}

In patients with left ventricular hypertrophy, coronary reserve is reduced due to an elevated basal coronary blood flow but maximal flow after dipyridamole infusion is within normal limits. Following aortic valve replacement, regression of left ventricular hypertrophy is accompanied by a decrease of resting coronary flow. Coronary flow reserve is improved because postoperative achieved maximal flow is essentially unchanged. Similarly coronary vasodilator capacity, assessed as coronary resistance reserve, is enhanced. Diastolic and systolic compressive forces, as well as structural changes of resistance vessels, seem to contribute little to altered coronary reserve. Preoperative left ventricular muscle mass and morphometric structure had no predictive value for postoperative vasodilator capacity.

\section{References}

[1] Heiss HW, Tauchert M, Strauer BE, Sonntag H, Kochsiek K. Koronare Hämodynamik und myokardialer Sauerstoffverbrauch bei Patienten mit linksventrikulärer Hypertrophie. Z Kreisl Forsch 1971; 61:260-70.

[2] Strauer BE. Myocardial oxygen consumption in chronic heart disease: role of wall stress, hypertrophy and coronary reserve. Am J Cardiol 1979; 44: 730-40.

[3] Tauchert M, Hilger HH. Application of coronary reserve concept to the study of myocardial perfusion. In: Schaper $W$, ed. The pathophysiology of myocardial perfusion. Amsterdam: Elsevier/North-Holland Biomedical Press, 1979: 141-67.
[4] Eastham CL, Doty DB, Hiratzka LF, Wright CB, Marcus ML. Volume-overload left ventricular hypertrophy impairs coronary reserve in humans. Circulation 1981; 64 (Suppl IV): IV-26 (Abstr).

[5] Hiratzka LF, Doty DB, Eastham CL, Mareus ML. Pressure versus volume overload has different effects on coronary reserve. Circulation 1982; 66 (Suppl II): II-354 (Abstr).

[6] Doty DB, Wright CB, Hiratzka LF, Eastham CL, Marcus ML. Coronary reserve in volume-induced right ventricular hypertrophy from atrial septum defect. Am J Cardiol 1984; 54: 1059-63.

[7] Opherk D, Mall G, Zebe Het al. Reduction of coronary reserve: a mechanism for angina pectoris in patients with arterial hypertension and normal coronary arteries. Circulation 1984; 69: $1-7$.

[8] Opherk D, Schwarz F, Manthey J, Kübler W. Coronary dilatory capacity in patients with severe aortic valve disease. Eur Heart J 1984; 5 (Suppl I): 207 (Abstr).

[9] Marcus ML, Doty DB, Hiratzka LF, Wright CB, Eastham CL. Decreased coronary reserve: a mechanism for angina pectoris in patients with aortic stenosis and normal coronary arteries. $\mathbf{N}$ Engl J Med 1982; 307: 1362-6.

[10] Nitenberg A, Foult JM, Antony I, Blanchent F, Rahali M. Coronary flow and resistance reserve in patients with chronic aortic regurgitation, angina pectoris and normal coronary arteries. J Am Coll Cardiol 1988; 11:478-86.

[11] Chilian WM, Marcus ML. Coronary vascular adaptations to myocardial hypertrophy. Ann Rev Physiol 1987; 49: 477-87.

[12] Marcus ML, Harrison DG, Chilian WM et al. Alterations in coronary circulation in hypertrophied ventricles. Circulation 1987; 75 (Suppl I): I 19-25.

[13] Müller TM, Marcus ML, Kerber RE, Young JA, Barnes RW, Abboud FM. Effect of renal hypertension and left ventricular hypertrophy on the coronary circulation in dogs. Circ Res 1978; 42: 543-9.

[14] Wangler RD, Peters KG, Marcus ML, Tomanek RJ. Effects of duration and severity of arterial hypertension and cardiac hypertrophy on coronary vasodilator reserve. Circ Res 1982; 51: $10-8$.

[15] Hoffman JIE. A critical view of coronary reserve. Circulation 1987; 75 (Suppl I): I 6-11.

[16] Stack RS, Schirmer B, Greenfield C. Coronary artery luminal diameters in normal and hypertrophied canine ventricles. Circulation 1980; 62 (Supp; III): III-64 (Abstr).

[17] Wicker P, Tarazi RC, Kobayashi K. Coronary blood flow during the development and regression of left ventricular hypertrophy in renovascular hypertensive rats. Am J Cardiol 1983; 51: 1744-9.

[18] Pantely G, Morton M, Rahimtoola SH. Effects of suocessful, uncomplicated valve replacement on ventricular hypertrophy, volume and performance in aortic stenosis and in aortic incompetence. J Thorac Cardiovasc Surg 1978; 75: 383-91.

[19] Krayenbuehl HP, Turina M, Hess OM, Rothlin M, Senning A. Pre- and postoperative left ventricular contractile function in patients with aortic valve disease. Br Heart J 1979; 41: 204-13.

[20] Dodge HT, Sandler H, Ballew DW, Lord JD. The use of biplane angiocardiography for the measurement of left ventricular volume in man. Am Heart J 1960; 60: 762-76.

[21] Rackley CE, Dodge HT, Coble YD, Hay RE. A method for determining left ventricular mass in man. Circulation 1964; 29: 666-71.

[22] Sandler $H$, Dodge HT. Left ventricular tension and stress in man. Cir Res 1963; 13: 91-104.

[23] Gaasch WH, Battle WE, Oboler AA, Banas JS, Levine HJ. Left ventricular stress and compliance in man with special reference to normalized ventricular function curves. Circulation 1972; 45: $746-62$.

[24] Ganz W, Tamura K, Marcus HS, Donoso R, Yoshida S, Swan HJC. Measurement of coronary sinus blood flow by continuous thermodilution in man. Circulation 1971; 44: 181-95.

[25] Mathey DG, Chatterjee K, Tyberg JV, Lekven J, Brundage B, Parmley WW. Coronary sinus reflux: A source of error in the measurement of thermodilution coronary sinus blood flow. Circulation 1978; 57: 778-86. 
[26] Strauer BE, Brune I, Schenk H, Knoll D, Perings E. Lupus cardiomyopathy: Cardiac mechanucs, hemodynamics, and coronary blood flow in uncomplicated systemic lupus erythematosus. Am Heart J 1976; 92: 715-22.

[27] Foult JM, Nitenberg A. Dipyridamole versus intracoronary injection of contrast medium for the evaluation of coronary reserve in man: a comparative study. Cathet Cardiovasc Diagn 1986; 12: 304-10

[28] Hess OM, Schneider J, Koch R, Bamert C, Grimm J, Krayenbuehl HP. Diastolic function and myocardial hypertrophy: special reference to normalized viscoelastic data. Circulation 1981; 63: 360-71.

[29] Weibel ER, Kistler GS, Scherle WF. Practical stereological methods for morphometric cytology. J Cell Biol 1966; 30: 23-38.

[30] Hess OM, Ritter M, Schneider J, Grimm J, Turina M, Krayenbuehl HP. Diastolic stiffness and myocardial structure in aortic valve disease before and after valve replacement. Circulation 1984; 69: 855-65.

[31] Alyono D, Anderson RW, Parrish DG, Dai XZ, Bache RJ Alterations of myocardial blood flow associated with experimental canine left ventricular hypertrophy secondary to valvular aortic stenosis. Circ Res 1986; 58: 47-57.

[32] Su-Fan Q, Brum J, Bove AA. Energy production, $\mathrm{O}_{2}$ consumption, and blood flow reserve in experimental aortic valve disease. Am J Physiol 1987; 252: H243-51.

[33] Marchetti GV, Merlo L, Noseda V, Visioli O. Myocardial blood flow in experimental cardiac hypertrophy in dogs. Circ Res 1973; 7: 519-27.

[34] Marcus ML, Müller TM, Eastham CL. Effects of short- and long-term ventricular hypertrophy on coronary circulation. Am J Physiol 1981; 241: H358-62.

[35] Kawachi K, Kawashima Y, Kitsamura S et al. Coronary sinus blood flow and myocardial oxygen consumption after valve replacement for aortic insufficiency. Ann Thorac Surg 1986; 42: $86-9$.
[36] O'Keefe DD, Hoffman JIE, Cheitlin R, O'Neill MJ, Allard JR, Shapkin E. Coronary blood flow in experimental canine left ventricular hypertrophy. Circ Res 1978; 43: 43-51.

[37] Harrison DG, Barnes DH, Hiratzka LF, Eastham CL, Kerber RE, Marcus ML. The effect of cardiac hypertrophy on the coronary collateral flow. Circulation 1985; 71: 1135-45.

[38] Scheel KW, Eisenstein BL, Ingram LA. Coronary, collateral, and perfusion territory responses to aortic banding. Am J Physiol 1984; 246: H768-75

[39] Doty DB, Eastham CL, Hiratzka LF, Wright CB, Marcus ML. Determination of coronary reserve in patients with supravalvular aortic stenosis. Circulation 1982; 66 (Suppl I): I 186-92.

[40] Tomanek RJ, Palmer PJ, Pieffer GW, Schreiber K, Eastham CL, Marcus ML. Morphometry of canine coronary arteries, arterioles, and capillaries during hypertension and left ventricular hypertrophy. Circ Res 1986; 58: 38-46.

[41] Marcus ML, Wilson RF, White CW. Methods of measurement of myyocardial blood flow in patients: a critical review. Circulation 1987; 76: 245-53.

[42] Rossen JD, Simonetti I, Marcus ML, Winniford MD. Coronary dilation with standard dose dipyridamole and dipyridamole combined with handgrip. Circulation 1989; 79: 566-72.

[43] Cannon RO, Schenke WH, Loon MB, Rosing DR, Urqhart J, Epstein SE. Limited coronary flow reserve after dipyridamole in patients with ergonovine-induced coronary vasoconstriction. Circulation 1987; 75: 163-74.

[44] Marchant E, Pichard A, Rodriguez JA, Casanegra P. Acute effect of systemic versus intracoronary dipyridamole on coronary circulation. Am J Cardiol 1986; 57: 1401-4.

[45] Cannon RO, Watson RM, Rosing DR, Epstein SE. Angina caused by reduced vasodilator reserve of the small coronary arteries. J Am Coll Cardiol 1983; 1: 1359-73.

[46] Bortone AS, Hess OM, Eberli FR et al. Abnormal coronary vasodilator syndrome in patients with normal coronary arteries and reduced coronary flow reserve. Circulation 1989; 79 ; 516-27. 